\title{
A note on moments of dividends
}

\author{
Hansjörg Albrecher*and Hans U. Gerber ${ }^{\dagger}$
}

\begin{abstract}
We reconsider a formula for arbitrary moments of expected discounted dividend payments in a spectrally negative Lévy risk model that was obtained in Renaud and Zhou [3] and in Kyprianou and Palmowski [4] and extend the result to stationary Markov processes that are skip-free upwards.
\end{abstract}

Keywords: dividends, barrier strategies, stationary Markov process, scale function.

2000 Mathematics Subject Classification: 91B30, 60G51, $62 \mathrm{P} 05$.

In two recent papers, Renaud and Zhou [3] and Kyprianou and Palmowski [4] independently showed that the $k$ th moment of the expected discounted dividend payments

${ }^{*}$ Professor of Actuarial Science, Faculty of Business and Economics, University of Lausanne, CH1015 Lausanne, Switzerland and Faculty Member of the Swiss Finance Institute. Support from the Swiss National Science Foundation Project 200021-124635/1 is acknowledged. e-mail: hansjoerg.albrecher@unil.ch

${ }^{\dagger}$ Distinguished Visiting Professor at the University of Hong Kong, and Honorary Professor of Actuarial Science, Faculty of Business and Economics, University of Lausanne, CH-1015 Lausanne, Switzerland, e-mail: hgerber@unil.ch 
in a spectrally negative Lévy risk model with initial surplus $u$ and horizontal dividend barrier $b \geq u$ is given by

$$
V_{k}(u ; b)=k ! \frac{W_{k \delta}(u)}{W_{k \delta}(b)} \prod_{i=1}^{k} \frac{W_{i \delta}(b)}{W_{i \delta}^{\prime}(b)} .
$$

Here $\delta \geq 0$ is a constant discount rate and $W_{q}(x)$ is the scale function of the underlying Lévy process (with Laplace exponent $\psi$ ) defined through its Laplace transform $\int_{0}^{\infty} e^{-\lambda x} W_{q}(x) \mathrm{d} x=1 /(\psi(\lambda)-q)$.

In this short note we show that formula (1) can be established in a more general framework by direct probabilistic reasoning. Concretely, assume that the surplus process $U(t)$ is a stationary Markov process that has no jumps upwards and has the strong Markov property. Let $T_{(0, a)}=\inf \{t \geq 0 \mid U(t) \notin(0, a)\}$ and define for $0 \leq u_{1} \leq u_{2}$ the function $C_{\delta}\left(u_{1}, u_{2}\right)=\mathbb{E}_{u_{1}}\left[e^{-\delta T_{\left(0, u_{2}\right)}} ; U\left(T_{\left(0, u_{2}\right)}\right)=u_{2}\right]$, which is the Laplace transform of the upper exit time out of the interval $\left(0, u_{2}\right)$ when starting in $u_{1}$, i.e. $U(0)=u_{1}$. As discussed in Gerber et al. [1], one immediately deduces from the absence of upward jumps and the strong Markov property that

$$
C_{\delta}\left(u_{1}, u_{3}\right)=C_{\delta}\left(u_{1}, u_{2}\right) C_{\delta}\left(u_{2}, u_{3}\right), \quad 0 \leq u_{1} \leq u_{2} \leq u_{3}
$$

Thus, there exists a positive increasing function $h_{\delta}(x)$ such that

$$
C_{\delta}\left(u_{1}, u_{2}\right)=\frac{h_{\delta}\left(u_{1}\right)}{h_{\delta}\left(u_{2}\right)} \quad \text { for } 0 \leq u_{1} \leq u_{2}
$$

(note that in the particular situation where $U(t)$ is a spectrally negative Lévy process, $h_{\delta}(x)$ can be identified with the scale function $\left.W_{\delta}(x)\right)$. Since the function $h_{\delta}(x)$ is unique only up to a constant factor, we can choose $u_{0}$ and set $h_{\delta}\left(u_{0}\right)=1$, giving

$$
h_{\delta}(u)= \begin{cases}C_{\delta}\left(u, u_{0}\right), & u<u_{0}, \\ 1 / C_{\delta}\left(u_{0}, u\right), & u>u_{0} .\end{cases}
$$

Dividends are paid according to the barrier strategy with horizontal barrier $b$, that is, any potential excess of the surplus beyond $b$ is paid as dividends. Let $D_{u}(t)$ denote the aggregate dividends paid up to time $t$, and let $\tau$ be the time of ruin. Then the present 
value of all dividends up to ruin is

$$
D_{u}=\int_{0}^{\tau} e^{-\delta t} \mathrm{~d} D_{u}(t)
$$

The $k$ th moment of $D_{u}$ is denoted by $V_{k}(u ; b)=\mathbb{E}_{u}\left(D_{u}^{k}\right)$.

Analogously to Proposition 2 of Renaud and Zhou [3], it immediately follows from $\left(e^{-\delta t} D_{u}\right)^{k}=e^{-k \delta t} D_{u}^{k}$ and the strong Markov property of $U(t)$ applied at the upper exit time of the interval $(0, b)$ that

$$
V_{k}(u ; b)=C_{k \delta}(u ; b) V_{k}(b ; b)=\frac{h_{k \delta}(u)}{h_{k \delta}(b)} V_{k}(b ; b), \quad 0 \leq u \leq b .
$$

Related to an idea of Gerber and Shiu [2], consider next the difference between the total discounted dividends when starting in $U(0)=b$ and $U(0)=b-\epsilon$, respectively, for a sufficiently small $\epsilon>0$. If $U(0)=b-\epsilon$, then the dividend barrier will be reached "shortly". At that time, the process that starts at $b$ has led to a total dividend of $\epsilon$, and after this time the trajectories of the two processes are identical. Hence we have the approximate relationship $D_{b}-D_{b-\epsilon} \approx \epsilon$ and subsequently $D_{b}^{k}-D_{b-\epsilon}^{k} \approx D_{b}^{k}-\left(D_{b}-\epsilon\right)^{k}=$ $\epsilon k D_{b}^{k-1}+o(\epsilon)$. Taking expectations and the limit $\epsilon \rightarrow 0$, we arrive at

$$
\left.\frac{\mathrm{d} V_{k}(u ; b)}{\mathrm{d} u}\right|_{u=b^{-}}=k V_{k-1}(b ; b) .
$$

From (2) and (3) we obtain the recursive formula

$$
V_{k}(b ; b)=k \frac{h_{k \delta}(b)}{h_{k \delta}^{\prime}(b)} V_{k-1}(b ; b) .
$$

From this and $V_{0}(u ; b)=1$ we obtain

$$
V_{k}(b ; b)=k ! \prod_{i=1}^{k} \frac{h_{i \delta}(b)}{h_{i \delta}^{\prime}(b)} .
$$

Substitution in (2) yields

$$
V_{k}(u ; b)=k ! \frac{h_{k \delta}(u)}{h_{k \delta}(b)} \prod_{i=1}^{k} \frac{h_{i \delta}(b)}{h_{i \delta}^{\prime}(b)}, \quad 0 \leq u \leq b,
$$

which extends (1) to stationary Markov processes that are skip-free upwards. 


\section{References}

[1] H.U. Gerber, S. Lin and H. Yang (2006) A note on the dividends-penalty identity and the optimal dividend barrier. Astin Bulletin 36(2), 489-503.

[2] H.U. Gerber and E.S.W. Shiu (2004) Reply to Discussions on "Optimal Dividends: Analysis with Brownian Motion". North American Actuarial Journal 8(2), 113-115.

[3] J. Renaud and X. Zhou (2007) Distribution of the present value of dividend payments in a Lévy risk model. Journal of Applied Probability 44, 420-427.

[4] A. Kyprianou and Z. Palmowski (2007) Distributional study of De Finetti's dividend problem for a general Lévy insurance risk process. Journal of Applied Probability 44, 428-443. 